Bio - grafia. Escritos sobre la Biología y su Enseñanza. ISSN 2027

Edición Extraordinaria. p.p. 437 - 447

Memorias del VIII Encuentro Nacional de Experiencias en Enseñanza de la Biología y la Educación Ambiental. III Congreso Nacional de Investigación en Enseñanza de la Biología.

\title{
Constitución y actividades de la Mesa Provincial de Educación Ambiental en la provincia de Mendoza, Argentina.
}

\section{Constitution and activities of the Provincial Bureau of Environmental Education in the province of Mendoza, Argentina.}

Diaz Isenrath, G.'; Bilbao, T.2; Barbosa, C.3: Lagos Silnik, S. 4; Marlia, N.5; Musso, S.6; Ongay, 0.7: Zárate, M. ${ }^{8}$; Pescetti, E. ${ }^{9}$; Buccheri, M. ${ }^{10}$; Bertolotti, F. ${ }^{11}$

\section{Resumen}

La Educación Ambiental como actividad interdisciplinaria e interinstitucional, requiere de la planificación estratégica participativa, que permita la concreción de esfuerzos que suelen realizarse de manera aislada. Se expone el proceso llevado a cabo para la constitución de la Mesa de Educación Ambiental en la provincia de Mendoza, Argentina. A partir de la discusión sobre la problemática local del Cambio Climático y los requerimientos de medidas de adaptación, se propone el espacio en el año 2013 mediante la firma de una declaración de interés entre 4 instituciones. En el año 2014 toma fuerza la iniciativa mediante la

1 . Facultad de Ciencias Exactas y Naturales - Universidad Nacional de Cuyo (UNCuyo).gdiaz@infoar.net 2 . Instituto Multidisciplinario de Ciencias Ambientales- UNCuyo. tania.ica@gmail.com 3 . Instituto Multidisciplinario de Ciencias Ambientales- UNCuyo. cristinabarbosa@yahoo.com.ar $4 \quad$. Instituto Argentino de Investigaciones de Zonas Áridas (IADIZA)- Consejo Nacional de Investigaciones Científicas y Técnicas (CONICET). slagos@mendoza-conicet.gob.ar 5 . Dirección General de Escuelas- Gobierno de Mendoza. noramarlia@yahoo.com.ar $6 \quad$. Facultad de Educación Elemental y Especial- UNCUYO. silviamusso@hotmail.com 7 . Ministerio de Tierra, Ambiente y Recursos Naturales-(MTAyRN). Gobierno de Mendoza. oscarongayugarteche@yahoo.com.ar

$8 \quad$.IADIZA - CONICET. zarate.mirta@gmail.com

9 . Universidad Tecnológica Nacional-CONICET. epescetti@mendoza-

conicet.gob.ar

10 . Instituto Nacional del Agua, Centro de Estudios y Legislación del Agua (INACELA).mauriciobucheri@yahoo.com.ar

.MTAyRN. Gobierno de Mendoza. florabertolotti@gmail.com 
Bio - grafia. Escritos sobre la Biología y su Enseñanza. ISSN 2027

Edición Extraordinaria. p.p. 437 - 447

Memorias del VIII Encuentro Nacional de Experiencias en Enseñanza de la Biología y la Educación Ambiental. III Congreso Nacional de Investigación en Enseñanza de la Biología.

definición de objetivos y metas concretas, con la participación activa más de 10 instituciones de diversos ámbitos: educativas, científicas, técnicas, órganos de gobierno, organizaciones no gubernamentales, municipios y privados. En el año 2015 se consolida mediante la firma de un Acta Constitutiva. Su objetivo principal es articular acciones que permitan a la comunidad tener mejores herramientas para enfrentar la complejidad de la problemática y el cuidado de nuestro ecosistema. Se considera un espacio proactivo y participativo, abierto a la incorporación de nuevos actores. Procura establecer lineamientos de políticas públicas sobre Educación Ambiental, orientadas a la promoción de comunidades sustentables. Asimismo, aspira a ser un órgano de articulación reconocido por ley. En su corto recorrido, ha avanzado en su organización interna, capacitaciones a docentes, sugerencias al Proyecto de Ley de Educación de la Provincia como la inclusión de la obligatoriedad de la enseñanza de la Educación Ambiental y la creación de la figura de referente ambiental en los establecimientos educativos, como así también organiza jornadas, concursos y eventos educativos a nivel provincial.

\section{Introducción}

La educación ambiental (EA) comienza a tener relevancia a partir de los años 70 , como respuesta a la crisis ambiental. El concepto de EA ha ido adecuándose al desarrollo del pensamiento ambiental desde una educación orientada a la preservación y conservación de la naturaleza; a la protección y mejoramiento del ambiente, y más recientemente al desarrollo sustentable. La preocupación surgida por el deterioro del planeta, es un síntoma de una crisis de nuestra civilización que cuestiona los vínculos sociedad-naturaleza. La EA es un campo de conocimiento dilemático y en construcción, en el que básicamente faltan acuerdos fundamentales sobre los conceptos de ambiente y sustentabilidad, que son los que orientan y determinan sus prácticas (Sauvé, 2009).

En Argentina con la Reforma de 1994 se incorpora el artículo 41 en nuestra Constitución Nacional, que crea el derecho a un ambiente sano, equilibrado y apto para el desarrollo humano, como así también impone a las autoridades la obligación de proveer a la información ambiental y EA. Se incluye también en la Ley 25675, "General de Política Ambiental Nacional" en los artículos 8, 14 y 15 como instrumentos de la política y la gestión ambiental.

Una política ambiental exitosa depende la conciencia ambiental ciudadana extendida y 
Bio - grafia. Escritos sobre la Biología y su Enseñanza. ISSN 2027

Edición Extraordinaria. p.p. 437 - 447

Memorias del VIII Encuentro Nacional de Experiencias en Enseñanza de la Biología y la Educación Ambiental. III Congreso Nacional de Investigación en Enseñanza de la Biología.

profunda en los individuos y los sectores sociales, y para alcanzarla, la EA debe convertirse en prioridad con amplia participación de todos los miembros de la comunidad.

Como proceso y acción a nivel mundial, latinoamericano, y nacional, la provincia de Mendoza posee experiencias de EA en diferentes ámbitos de la sociedad. El presente trabajo pretende comenzar con el proceso de recopilación de las mismas, que no pretende ser exhaustiva. Éstas son la base sobre la que se construye la Mesa Provincial de Educación Ambiental (MPEA). Ésta surge como necesidad de integrar los esfuerzos aislados y muchas veces sin continuidad, como así también articular instituciones, mediante un abordaje transdisciplinar y de colaboración intersectorial.

\section{Antecedentes de Educación Ambiental en Mendoza}

En nuestra provincia la Ley 5961 "Preservación del Medio Ambiente y territorio de Mendoza", posee un capítulo dedicado a la EA. En el mismo sentido también en la Ley 6045 de "Áreas Protegidas" en su Capítulo II, artículo 5to, la Ley 7874 de "Protección del Arbolado Público", en su artículo 39, y la Ley 8051 de "Ordenamiento Territorial y usos del Suelo" que en su artículo $4^{\circ}$, mencionan la EA.

Los hábitos, valores y acciones que supone esta dimensión social son objeto de enseñanza y de aprendizaje tanto en los ámbitos formales como no formales de la educación. Palabras como recursos naturales, contextos, problemas, cambios y conflictos ambientales, intencionalidades y acciones sociales, procesos y ambiente, están presentes en las agendas

de muchas acciones concretas que se llevan a cabo en Mendoza. Éstas son numerosas y todas ellas se organizan alrededor de ejes estratégicos como prevenir, promover, remediar, fortalecer e impulsar y convergen en el abordaje de las siguientes problemáticas: desertización y desertificación, pérdida de biodiversidad,invasiones biológicas, comercio ilegal de especies y caza furtiva, cambio climático, degradación de áreas naturales y bosques nativos, disminución del arbolado público y espacios verdes urbanos, metropolización y concentración poblacional, entre otras.

\subsection{Educación Formal}

La Ley Federal de Educación de 1993 y la Constitución de 1994 se hace referencia a la Educación Ambiental como tema transversal, con un enfoque que recibe el aporte de diferentes campos de conocimiento, pero no como disciplina independiente (Tello y Pardo, 1996). En el año 2009 la ley N²6.206 Ley de Educación Nacional en su artículo 89 expresa 
Bio - grafia. Escritos sobre la Biología y su Enseñanza. ISSN 2027

Edición Extraordinaria. p.p. 437 - 447

Memorias del VIII Encuentro Nacional de Experiencias en Enseñanza de la Biología y la Educación Ambiental. III Congreso Nacional de Investigación en Enseñanza de la Biología.

que el Ministerio de Educación, Ciencia y Tecnología, en acuerdo con el Consejo Federal de Educación, dispondrá las medidas necesarias para proveer la EA en todos los niveles y modalidades del Sistema Educativo Nacional, con la finalidad de promover valores, comportamientos y actitudes que sean acordes con un ambiente equilibrado y la protección de la diversidad biológica; que propendan a la preservación de los recursos naturales y a su utilización sostenible y que mejoren la calidad de vida de la población. A tal efecto se definirán en dicho ámbito institucional, utilizando el mecanismo de coordinación que establece el artículo 15 de la Ley $N^{\circ} 25.675$, las políticas y estrategias destinadas a incluir la EA en los contenidos curriculares comunes y núcleos de aprendizaje prioritario, así como a capacitar a los/as docentes en esta temática.

Acorde a esta, actualmente en los diseños curriculares de la provincia de Mendoza se observa una actitud favorable hacia la presencia de la EA en los diseños curriculares de todos los niveles: Documento Curricular Provincial (DCP) de Nivel Inicial, los Saberes Indispensables de nivel primario, el DCP de Secundaria Orientada y Diseño Curricular del Profesorado de Biología, del Profesorado de Educación Primaria, y Tecnicatura en Conservación de la Naturaleza. En otras carreras relacionadas a temas ambientales como Gestión Ambiental, Gestión de Recursos Hídricos, Economía Social y Desarrollo Local, la EA no se encuentra como espacio curricular sino como contenido dentro de otros como Legislación Ambiental, o Problemática Ambiental.

Tanto universidades públicas como privadas, han avanzado en la incorporación de la temática ambiental, desde distintas miradas disciplinares, como la Ingeniería en Recursos Naturales, Licenciatura en Ciencias Básicas, orientación Biología y Química de la Facultad de Ciencias Exactas y Naturales de la Universidad Nacional de Cuyo (UNCuyo), Licenciatura en Gestión Ambiental de la Universidad de Congreso, Licenciatura en Ecología de la Universidad Champagnat, etc. Durante la primer década de 2000 se implementó en la Facultad de Educación Elemental y Especial de la UNCuyo la Licenciatura en Educación Ambiental. Actualmente se ofrece la Maestría en Gestión Integrada de los Recursos Hídricos (Universidad Nacional de Córdoba, UNCuyo y Universidad Nacional del Litoral), la Maestría en Ingeniería Ambiental en la UNCuyo y la formación para el Ordenamiento Territorial en la Facultad de Filosofía y Letras de la UNCuyo. Existen además diferentes institutos y centros de investigación relacionado a temas ambientales. Muchos de ellos poseen programas de divulgación científica. En el Instituto Argentino de Investigaciones de Zonas Áridas (IADIZA) dependiente del Consejo Nacional de Investigaciones 
Bio - grafia. Escritos sobre la Biología y su Enseñanza. ISSN 2027

Edición Extraordinaria. p.p. 437 - 447

Memorias del VIII Encuentro Nacional de Experiencias en Enseñanza de la Biología y la Educación Ambiental. III Congreso Nacional de Investigación en Enseñanza de la Biología.

Científicas y Técnicas en el año 1996 formó el grupo de transferencia educativa, que favorece la capacitación en EA del docente, atendiendo las demandas en contenidos curriculares, aspectos metodológicos e institucionales. Otros casos a mencionar son el Instituto Nacional del Agua, Centro de Estudios y Legislación del Agua con su programa de capacitación sobre recursos hídricos, el Departamento General de Irrigación a través del Programa Ciudadanía del Agua.

Desde la Dirección General de Escuelas, la oferta de capacitación virtual es abundante, y gran parte de ella centrada en el uso de las TIC. Otras instituciones han ofrecido capacitaciones virtuales para docentes como IADIZA, UNCuyo, Universidad Tecnológica Nacional, etc. LaEA es abordada en los mismos desde diferentes enfoques.

Desde el Portal Educativo de la provincia de Mendoza se ha realizado en los últimos años un importante esfuerzo de recopilación y difusión de recursos muy valiosos para docentes y estudiantes. Muchos de ellos han sido elaborados por diferentes instituciones. La producción de estos materiales (publicaciones periódicas, libros, afiches, folletos) es muy variada.

Además, se ofrecen actividades para estudiantes y docentes, como senderos educativos como El Sendero del Garabato en la Cuenca Aluvional Piloto y los de las distintas áreas naturales protegidas.

\subsection{Educación no Formal}

Más allá de la educación formal, existen actualmente diversas organizaciones de la sociedad civil y organizaciones no gubernamentales, abocadas a difundir, concientizar, investigar y gestionar temáticas vinculadas a la EA en Mendoza. Muchas de ellas articulan con el ámbito formal e instalan en la agenda debates y temáticas ambientales de relevancia local. Entre ellas podemos mencionar Fundación Villavicencio, OIKOS Red Ambiental, Fundación Cullunche, Asambleas mendocinas por el agua, Fundación INVESCIENCIAS, BIOTA.

Asimismo se destaca el aporte de Centros Culturales, Clubes, Uniones Vecinales, entre otras, a la construcción de comunidades sustentables. Las experiencias no formales de capacitación y promoción en materia ambiental han logrado un protagonismo de gran impulso en Mendoza.

La EA ha surgido y cobrado fuerza en los distintos niveles de gobierno, considerándola en nuestros días una herramienta indispensable que acompaña a la gestión ambiental y 
Bio - grafia. Escritos sobre la Biología y su Enseñanza. ISSN 2027

Edición Extraordinaria. p.p. 437 - 447

Memorias del VIII Encuentro Nacional de Experiencias en Enseñanza de la Biología y la Educación Ambiental. III Congreso Nacional de Investigación en Enseñanza de la Biología.

contribuye a que ésta alcance con éxito sus objetivos.

En el año 1989 se crea el Ministerio de Ambiente, Urbanismo y Vivienda en Mendoza, el mismo desarrolló las primeras acciones de EA en la Provincia. En este ámbito, en el año 1998 se elabora el Primer Manual sobre EA de Mendoza. Posteriormente, en el año 2004 se efectiviza el Primer Programa Provincial de EA (Blanc, 2004), el cual se desarrolla actualmente bajo la dependencia de la Dirección de Recursos Naturales RenovablesMinisterio de Tierra Ambiente y Recursos Naturales. Actualmente trabaja de manera articulada con otras organizaciones en las capacitaciones de "Inspector ambiental", fortalecimiento de la Policía Ambiental, formación de monitores y promotores ambientales, capacitaciones en conservación de la naturaleza (estrechamente relacionada a áreas naturales protegidas), concursos, efemérides ambientales, y elaboración de material didáctico de EA.

En el ámbito gubernamental, el Área Educativa del Zoológico de Mendoza, trabaja para promover cambios de actitudes frente a la biodiversidad, el mascotismo, etc. a través de charlas, talleres, visitas, obras de títeres.

Los Municipios de la provincia, a partir de la década de los 90 comienzan a ser receptores de los reclamos ambientales, pero recién a partir del año 2000 comienzan a crearse dependencias dedicadas para recibirlos. Los municipios son encargados del tratamiento de residuos y el mantenimiento y conservación del arbolado público. Incluso algunos están creando reservas municipales. En los últimos años, la EA está acompañando a la gestión, y se han incorporado, programas de EA, capacitaciones, campañas de difusión y más recientemente se han sancionado ordenanzas que promueven la EA. Muchas de estas actividades llevadas a cabo conjuntamente con escuelas, centros culturales, clubes y uniones vecinales.

El Ente Provincial de Agua y Saneamiento, creado en 1993 como organismo autárquico del estado provincial, es encargado del Control, Regulación y Defensa de los Derechos del Usuario del Sistema Sanitario de Mendoza. Ha implementado distintas campañas de concientización y brinda en su plataforma virtual información útil destinada a estudiantes, sobre el cuidado de los recursos hídricos.

\section{Mesa Provincial de Educación Ambiental (MPEA)}

En el marco de la discusión sobre la problemática local del Cambio Climático y los requerimientos de medidas de adaptación, se realiza en el año 2013, un curso de 
Bio - grafia. Escritos sobre la Biología y su Enseñanza. ISSN 2027

Edición Extraordinaria. p.p. 437 - 447

Memorias del VIII Encuentro Nacional de Experiencias en Enseñanza de la Biología y la Educación Ambiental. III Congreso Nacional de Investigación en Enseñanza de la Biología.

capacitaciones para educadores sobre Cambio Climático en Mendoza "Cuyún, Cambia tus hábitos, no el Clima", iniciativa llevada adelante por el Instituto de Ciencias Ambientales y la Facultad de Educación Elemental y Especial, ambos de la Universidad Nacional de Cuyo, en conjunto con la Dirección General de Escuelas, la entonces Secretaría de Medio Ambiente, y la Municipalidad de Malargüe.

Rescatando el carácter de colaboración interinstitucional y enfoque multidisciplinar del curso, las autoridades presentes firmaron durante el acto de inauguración, la Declaración de Interés para la conformación de una Mesa Provincial de Educación Ambiental. En julio del año 2014 toma fuerza nuevamente la iniciativa durante el "1 ${ }^{\circ}$ Encuentro sobre Cambio Climático", organizado por el Ministerio de Tierra Ambiente y Recursos Naturales. A través de una mesa de trabajo sobre EA ante el Cambio Climático, se retoma el debate sobre la necesidad de articular esfuerzos y experiencias en pos de la EA en Mendoza. Allí se incorporan nuevas instituciones de diversos ámbitos: educativo, científico, técnico, órganos de gobierno, organizaciones de la sociedad civil, municipios y sector privado. Desde entonces la MPEA adquiere mayor institucionalidad y se establecen reuniones formales, con frecuencias regulares, dejando constancia en actas, en las cuales se fueron estableciendo objetivos comunes y acciones concretas a realizar.

A fines del año 2014, encuadrado en el debate por la aprobación del Ley de Educación Provincial, la MPEA presenta ante las Cámaras Legislativas la propuesta de incorporar la EA como un espacio curricular transversal y la creación de la figura de un "referente ambiental", responsable de articular actividades ambientales en cada unidad educativa.

En el Día Mundial del Ambiente, 5 de junio del año 2015, la MPEA se consolida mediante la firma de un Acta Compromiso, firmada por autoridades de más de 15 instituciones de diversos ámbitos.

Participan actualmente de la MPEA representantes del Gobierno de Mendoza: Ministerio de Tierras, Ambiente y Recursos Naturales, Dirección General de Escuelas, Municipalidad de Malargüe, Municipalidad de Luján de Cuyo, Municipalidad de Godoy Cruz, Ministerio de Turismo; de la Universidad Nacional de Cuyo: Secretaría de Desarrollo InstitucionalInstituto de Ciencias Ambientales, Facultad de Educación Elemental y Especial Facultad de Filosofía y Letras, Facultad de Ciencias Exactas y Naturales, Facultad de Ciencias Agrarias, Facultad de Ciencias Médicas, Facultad de Ciencias Políticas y Sociales; del Centro Científico Tecnológico del Consejo Nacional de Investigaciones Científicas y Técnicas: IADIZA e Instituto Argentino de Nivología, Glaciología y Ciencias Ambientales; 
Bio - grafia. Escritos sobre la Biología y su Enseñanza. ISSN 2027

Edición Extraordinaria. p.p. 437 - 447

Memorias del VIII Encuentro Nacional de Experiencias en Enseñanza de la Biología y la Educación Ambiental. III Congreso Nacional de Investigación en Enseñanza de la Biología.

de la Universidad Tecnológica Nacional: Facultad Regional Mendoza; Universidad de Congreso: Instituto Superior Técnico de Estudios Económicos, Fundación Villavicencio, Fundación INVECIENCIAS.

Como menciona el Acta de Compromiso, se trata de "..un espacio intersectorial proactivo y participativo, abierto a la incorporación de nuevos actores, que establece las bases de colaboración, cooperación y asesoramiento para el apoyo de las temáticas relacionadas al conocimiento, gestión, educación y políticas integrales de la Educación Ambiental, incluidas en las currículas de todos los niveles educativos en ámbitos formales y no formales","...pretendiendo que este espacio se convierta en una plataforma en la que se traten: temáticas ambientales locales vinculadas al estudio y comprensión de los diferentes ambientes de la Provincia de Mendoza; estudio, promoción y uso de energías renovables; gestión integral de residuos, gestión del hábitat sustentable; promoción de la participación ciudadana, de la salud, entre otras, con el fin de que las organizaciones de ciencia, tecnología, educación y gestión públicas y privadas encuentren en este espacio las estrategias para impulsar la adopción de lineamientos de políticas públicas de Educación Ambiental orientadas a la promoción de comunidades sustentables." (Barbosa et al, 2015). En este sentido, la MPEA, se configura desde sus inicios como un espacio que promueve la amplia participación, que diseña y ejecuta planes sobre EA, que busca integrar visiones provenientes de ámbitos diversos, de conciliar miradas, diferentes, que garanticen acuerdos de trabajo, y construcción de principios realizables. Se destaca el trabajo cooperativo, el respeto, la búsqueda de valores comunes y el reconocimiento de la riqueza y diversidad de los esfuerzos que antecedieron su creación. Pretende establecer espacios de encuentro, reflexión e intercambio de ideas sobre la EA en nuestro contexto local, que permitan unir esfuerzos y dar continuidad a las iniciativas exitosas, evitando enfoques catastróficos y extrapolados de otras realidades. Su objetivo principal es articular acciones entre sus actores, que permitan a la comunidad tener mayores herramientas para enfrentar la complejidad en el cuidado de nuestro territorio. Asimismo, aspira a ser un órgano de articulación reconocido por ley. Se propone promover a la EA como Política Pública que fortalezca la gestión estatal y la construcción de comunidades sustentables; incorporar la temática ambiental en todos los niveles de enseñanza y de modo transversal; capacitar a los docentes y educadores, como agentes multiplicadores de la educación formal y no formal.

La MPEA se está organizando en comisiones y tiene establecidos encuentros quincenales, 
Bio - grafia. Escritos sobre la Biología y su Enseñanza. ISSN 2027

Edición Extraordinaria. p.p. 437 - 447

Memorias del VIII Encuentro Nacional de Experiencias en Enseñanza de la Biología y la Educación Ambiental. III Congreso Nacional de Investigación en Enseñanza de la Biología.

con metas y objetivos a corto, mediano y largo plazo.

A corto plazo, se ha propuesto la construcción de un sitio web que permita concentrar la información disponible en formato digital, accesible tanto para docentes como para la comunidad en general. Por otro lado, se organizó un primer evento común denominado "Conciencia Ambiental", en referencia el Día de la Conciencia Ambiental, 26 de setiembre, en el cual se convoca la participación de niños, jóvenes y educadores a través de expresiones artísticas e intercambio de experiencias.

La MPEA aspira a consolidarse con la adopción de un plan de trabajo anual consensuado entre las instituciones miembro; recopilar y sistematizar experiencias locales; desarrollar propuestas de estudios y capacitaciones sobre EA; la elaboración de material educativo destinado a los ámbitos formales y no formales, la promoción de instancias de formación superior sobre la temática especifica.

\section{Reflexiones y desafios}

Si bien, las instituciones y experiencias mencionadas no son suficientes, las hemos tratado de capitalizar para comenzar a registrar los avances logrados en la EA de la provincia de Mendoza. Estos nos permiten esbozar algunas reflexiones críticas y comenzar a explicitar algunos desafíos ante los que nos encontramos.

En la Provincia de Mendoza las experiencias respecto a la EA no han sido continuas ni sistematizadas, en gran parte esto se debe al cambio de funcionarios producto de cambios de Gobiernos. De esta manera, aquellas experiencias exitosas sólo podemos rastrearla en la memoria de quienes las llevaron a cabo. Hoy, la MPEA, en una propuesta que busca paliar aquellas falencias y constituirse en un actor clave para el diseño y ejecución de las políticas públicas vinculadas a la EA.

Por un lado, hay temáticas que están más difundidas, y donde ya existe un conocimiento público como es el agua, los residuos y la contaminación. Esto no significa, que los hábitos cambien. Conocer sobre el problema es sólo un paso. Otros temas son más ajenos a la mayoría, como el uso de energías renovables y biodiversidad. Aunque este último ha recibido mucha atención por parte de diferentes organizaciones (IADIZA, Dirección de Recursos Naturales Renovables, Zoológico, Cullunche, Biota, etc.) su discusión sigue estando restringido a ciertos círculos o ambientes. En general las experiencias son muy diversas en el público, calidad de la información, tipo de recursos que utiliza y grado de profundidad. Queda pendiente la inclusión de iniciativas y experiencias desde los medios de 
Bio - grafia. Escritos sobre la Biología y su Enseñanza. ISSN 2027

Edición Extraordinaria. p.p. 437 - 447

Memorias del VIII Encuentro Nacional de Experiencias en Enseñanza de la Biología y la Educación Ambiental. III Congreso Nacional de Investigación en Enseñanza de la Biología.

comunicación y emprendimientos privados.

Por otro lado, la educación formal se vale de abundante teoría, y formas sistemáticas de recopilación de información, lo cual, ante la EA no formal, la posiciona en un lugar de privilegio. Lograr un justo equilibrio entre ambas, requiere recuperar y valorar la diversidad de ámbitos educativos no formales, como lo son municipios, áreas protegidas, organizaciones sociales, etc. donde surgen iniciativas desde las realidades cotidianas.

Desde las instituciones, puede observarse una creciente tendencia a la incorporación de la EA como instrumento de educación, comunicación y transferencia. Sin embargo, queda pendiente conocer cuál es la percepción de las personas "puertas adentro" de cada institución. Asimismo, en muchos casos se logra la convergencia de los enfoques natural, social, cultural, económico y político, en otros, todavía se percibe la EA como procedente de las ciencias naturales.

En los inicios del proceso de constitución de la MPEA, comenzamos a vislumbrar algunos desafíos, que podemos resumirlos de la siguiente manera:

- Lograr la articulación de sectores diversos, encuadrados en un espacio formal, que requiere apoyo institucional y sostenido en el tiempo, más allá de los compromisos y voluntad de sus integrantes, y los cambios de gestión, con su variabilidad en apoyo presupuestario, prioridades en la agenda, disponibilidad de recurso humano capacitado, entre otros elementos que limitan la continuidad de acciones y esfuerzos.

Proponer una nueva forma de recrear las políticas públicas, desde la reflexión participativa para, de esta manera, contribuir a la construcción de una ciudadanía comprometida con la calidad ambiental.

- Fortalecer la EA en todos los niveles de la educación formal y recuperar el valor de las experiencias en el ámbito no formal

- Lograr el funcionamiento y reconocimiento de la MPEA en Mendoza.

\section{Agradecimientos}

Agradecemos especialmente a quienes nos brindaron información, Pia Santarelli de la Municipalidad de Luján de Cuyo, Lourdes Vargas de la Municipalidad de Godoy Cruz, Laura Álvarez Di Paolo de la Municipalidad de Malargüe, y a Stella Fosarolli de la Dirección de Turismo Mendoza. Asimismo, a todos los integrantes de la MPEA que participan y se están sumando, para construir juntos este nuevo espacio. 
Bio - grafia. Escritos sobre la Biología y su Enseñanza. ISSN 2027

Edición Extraordinaria. p.p. 437 - 447

Memorias del VIII Encuentro Nacional de Experiencias en Enseñanza de la Biología y la Educación Ambiental. III Congreso Nacional de Investigación en Enseñanza de la Biología.

\section{Bibliografía}

Barbosa C, T. Bilbao, G. Diaz Isenrath, F. Bertolotti, S. Lagos Silnik, N. Marlia, S. Musso; O. Ongay, M. Zárate, E. Pescetti \& M. Buccheri (2015). Constitución de la Mesa Provincial de Educación Ambiental. Reformas institucionales y Educación Ambiental en la currícula escolar de Mendoza" XII Congreso Nacional de Ciencia Política. La Política en balance: debates y desafíos regionales. Mendoza.

Blanc, G. (2004). Proyecto de Educación Ambiental para la Provincia de Mendoza, presentado ante el Ministerio de Ambiente y Obras Públicas del Gobierno de Mendoza, Mza. En: Memorias del V Congreso Internacional de Educación Ambiental, La Habana, Cuba, 2005.

Ministerio de Ambiente y Obras Públicas de Mendoza (1998). Manual de Educación ambiental de Mendoza. MAOP, Mendoza, Argentina.

Sauvé, L. (1999). La educación ambiental entre la modernidad y la posmodernidad: en busca de un marco de referencia educativo integrador. Tópicos en educación ambiental 1 (2), 7 25.

Tello, B. \& A. Pardo (1996). Presencia de la Educación Ambiental en el nivel medio de enseñanza de los países iberoamericanos. Revista Iberoamericana de Educación 11. Educación ambiental: teoría y práctica. 